\title{
LIPOPROTEIN PATTERN AS A FUNCTION OF TOTAL TRIGLYCERIDE CONCENTRATION OF SERUM *
}

\author{
By MARGARET J. ALBRINK \\ (From the Department of Internal Medicine, Yale University School of Medicine, and the \\ Medical Service of the Grace-New Haven Community Hospital, \\ New Haven, Conn.)
}

(Submitted for publication July 21, 1960; accepted October 27, 1960)

The occasional occurrence of lactescent or milky serum in patients with certain diseases caused interest and speculation at least as long ago as 1799 when Mariet described turbid sera in some patients with diabetic acidosis (1). The lightscattering fat particles or chylomicrons causing lactescence are present in modest numbers after a fat meal but become scarce in normal postabsorptive sera. In certain pathological states, however, chylomicrons occur in excessive numbers, giving rise to a characteristic milky appearance of the sera.

In a previous study, lactescence was reported to be directly proportional to the total triglyceride concentration in serum (2). Turbidity was invariably present when total triglyceride fatty acid concentration exceeded $20 \mathrm{mEq}$ per L. As triglyceride concentration increased further, a progressively greater proportion of cholesterol and phospholipids also occurred in the particulate lipid fraction which could be readily removed by flotation of unaltered serum at moderate speeds in the ultracentrifuge.

Serum lipids are now known to exist in a spectrum of lipoproteins of varying density, from very low density particulate chylomicrons composed chiefly of triglycerides, through various classes of low density lipoproteins relatively rich in cholesterol, to high density lipoproteins in which phospholipids are prominent (3).

Interest in various classes of low density lipoproteins has been stimulated because of their possible role in the etiology of coronary arterial disease (4). Studies from this laboratory (5-7) have shown serum triglyceride concentration to

* Supported (in part) by Grant H-3498(C2) from the National Heart Institute, Bethesda, Md., and by a grant from The James Hudson Brown Memorial Fund. This work was done during tenure of an established investigatorship of the American Heart Association. be intimately associated with this disease. Antonis and Bersohn have also found elevation of serum triglycerides in ischemic heart disease (8). From the known composition of very low density lipoproteins, it might be inferred that their presence in abnormally high concentrations would be associated with increased concentration of total serum triglycerides.

The present study was undertaken to learn whether total triglyceride concentration might determine not only the fraction of lipids present in chylomicrons but also the partition of lipids among the various low density lipoproteins. At the same time the lipoprotein composition of the relatively clear subnatant fluid after removal of the chylomicron "cream" layer of lactescent sera could be determined. This clear fraction was previously analyzed in toto (2), but no analysis of lipoproteins was made.

\section{METHODS}

Serum was obtained from medical school personnel without known disease and from patients in the wards and clinics of the Grace-New Haven Community Hospital. Markedly lactescent sera were obtained from patients with essential hyperlipemia, tuberous xanthomata, or diabetic acidosis. Slightly or moderately lipemic sera were found in patients who had suffered a myocardial infarction but who had none of the stigmata of hyperlipemia. One coronary patient had normal lipids. Patients with hypothyroidism or with essential hypercholesterolemic xanthomatosis provided the sera with high cholesterol but normal triglycerides. The subjects and patients, all of whom were studied in the postabsorptive state, are listed in Table I, together with the serum lipid data and a brief clinical identification.

Blood was drawn from an antecubital vein and the cells separated from the serum within 2 hours of the venipuncture. The serum was subjected to 4 successive centrifugations, in the no. 40 rotor of a Spinco model L preparative ultracentrifuge, with removal of various fractions in the following manner.

1. Unaltered serum was centrifuged at $18,000 \mathrm{rpm}$ $(20,000 \mathrm{G})$ for 1 hour in order to remove most of the 
particulate lipids, chiefly chylomicrons. The bottom one-half to two-thirds of the clear subnatant fluid was withdrawn through a needle into a syringe; an aliquot was taken for analysis. The chylomicron composition was calculated by subtracting the concentration of lipid components in this layer from their concentration in the original serum. The assumption was made that no significant migration of lipoprotein other than chylomicrons occurred as a result of such mild ultracentrifugation (2). In very lipemic sera, a slight opalescence remained in the subnatant fraction indicating that not all visible lipid particles had been removed. Therefore, for want of a better term, the lipids removed by this first centrifugation, previously referred to as "insoluble lipids" or "emulsified lipids" (2), will here be called "light" chylomicrons.

2. The subnatant serum (usually $10 \mathrm{ml}$ ) obtained by Procedure 1 was, without further alteration, centrifuged at $40,000 \mathrm{rpm}(105,000 \mathrm{G})$ for 22 hours. A clear separation between the supernatant and subnatant lipids was marked by a water-clear middle zone. A Spinco tube slicer was used to slice the tubes through the clear zone at the junction of the upper and middle thirds. The entire supernatant fraction was carefully transferred to a $5 \mathrm{ml}$ volumetric flask with several washings of physiological saline, made to volume, and analyzed for lipids. This layer was markedly lipemic when the original serum was lipemic. The lipids so isolated are designated "heavy" chylomicrons, although it is recognized that they represent a transitional stage between chylomicrons and denser lipoproteins. Assuming the nonprotein solvent density of serum to be 1.006 (9), the "light" chylomicrons plus the "heavy" chylomicrons together constitute the fraction of lipids of density less than 1.0006 and are designated as $\mathrm{D}<1.006$.

3. The subnatant solution from Procedure 2 was transferred to a $10 \mathrm{ml}$ volumetric flask, with several washings of physiological saline. This was adjusted to D 1.019 according to the method of Havel, Eder and Bragdon (9), and spun at $40,000 \mathrm{rpm}$ for 22 hours in the ultracentrifuge $(105,000 \mathrm{G})$. The top third of the fluid was removed as in step 2 and analyzed. This fraction contained lipoproteins D 1.006-1.019.

4. The subnatant fluid was removed as in step 3, adjusted to density 1.063 by the method of Havel and coworkers, and centrifuged at $40,000 \mathrm{rpm}$ for 22 hours. The top third of the fluid, constituting the lipoproteins of D 1.019-1.063, was removed for analysis. The subnatant fluid was also analyzed and designated as fraction $\mathrm{D}>1.063$. During all centrifugations the centrifuge chamber was refrigerated in order to maintain the temperature of the sera below $18^{\circ} \mathrm{C}$.

The original serum and each of the fractions were extracted with 2:1 (vol/vol) chloroform-methanol. Water was added to create a two-phase system and the chloroform phase was analyzed for total fatty acids (10), for total cholesterol by the method of Abell, Levy, Brodie and Kendall (11), and for lipid phosphorus by a modification of the method of Fiske and Subbarow (12). The tri- glycerides were calculated as the difference between total fatty acids and fatty acids of cholesterol esters and phospholipids, using assumptions previously described (10). Triglycerides were expressed as milliequivalents per liter of triglyceride fatty acid. Since free cholesterol was not determined, it was assumed that 72 per cent of the cholesterol was esterified. The known differences in the ratio of free to total cholesterol in different lipoproteins (3) would introduce only a small error in the estimation of triglycerides. The triglycerides so calculated include the free fatty acids, which may account for appreciable amounts of the triglycerides in the fraction of density $>1.063$. The concentration of components of each density fraction was expressed in terms of its concentration in whole undiluted serum.

\section{RESULTS}

The concentration of triglyceride fatty acids, cholesterol and the cholesterol-phospholipid ratios of each serum are shown in Table I. The sera are listed in order of increasing triglyceride concentration. A triglyceride concentration greater than $5.5 \mathrm{mEq}$ per $\mathrm{L}$ is considered the upper limit of normal by the standards of this laboratory $(6$, $7,13)$. A brief clinical description of each subject is given. Except for E. E., who was studied twice, each serum was obtained from a different person.

The lipid concentration of the uncentrifuged sera varied greatly. The triglyceride fatty acid concentration varied from 2.1 to $200 \mathrm{mEq}$ per L, and the cholesterol from 179 to 792 per $100 \mathrm{ml}$. The cholesterol of whole serum tended to increase as the triglyceride increased, but with a great deal of variation. In some instances cholesterol was markedly elevated in the presence of normal triglycerides and in others was normal in the presence of somewhat increased concentrations of triglycerides. The cholesterol to phospholipid ratio varied from 0.71 to 1.33 . As previously noted (2), slight to moderate lactescence appeared as the triglyceride fatty acid concentration approached $20 \mathrm{mEq}$ per $\mathrm{L}$, and became marked when triglycerides exceeded this concentration.

The total concentration of lipids recovered in the lipoprotein fractions varied from 79 to 95 per cent of their concentration in the original serum, the incomplete recovery resulting most likely from the summation of small losses frequently encountered through leakage around the caps of the lustroid tubes in which the fractions were centrifuged. The losses affected total fatty acid, cholesterol and 


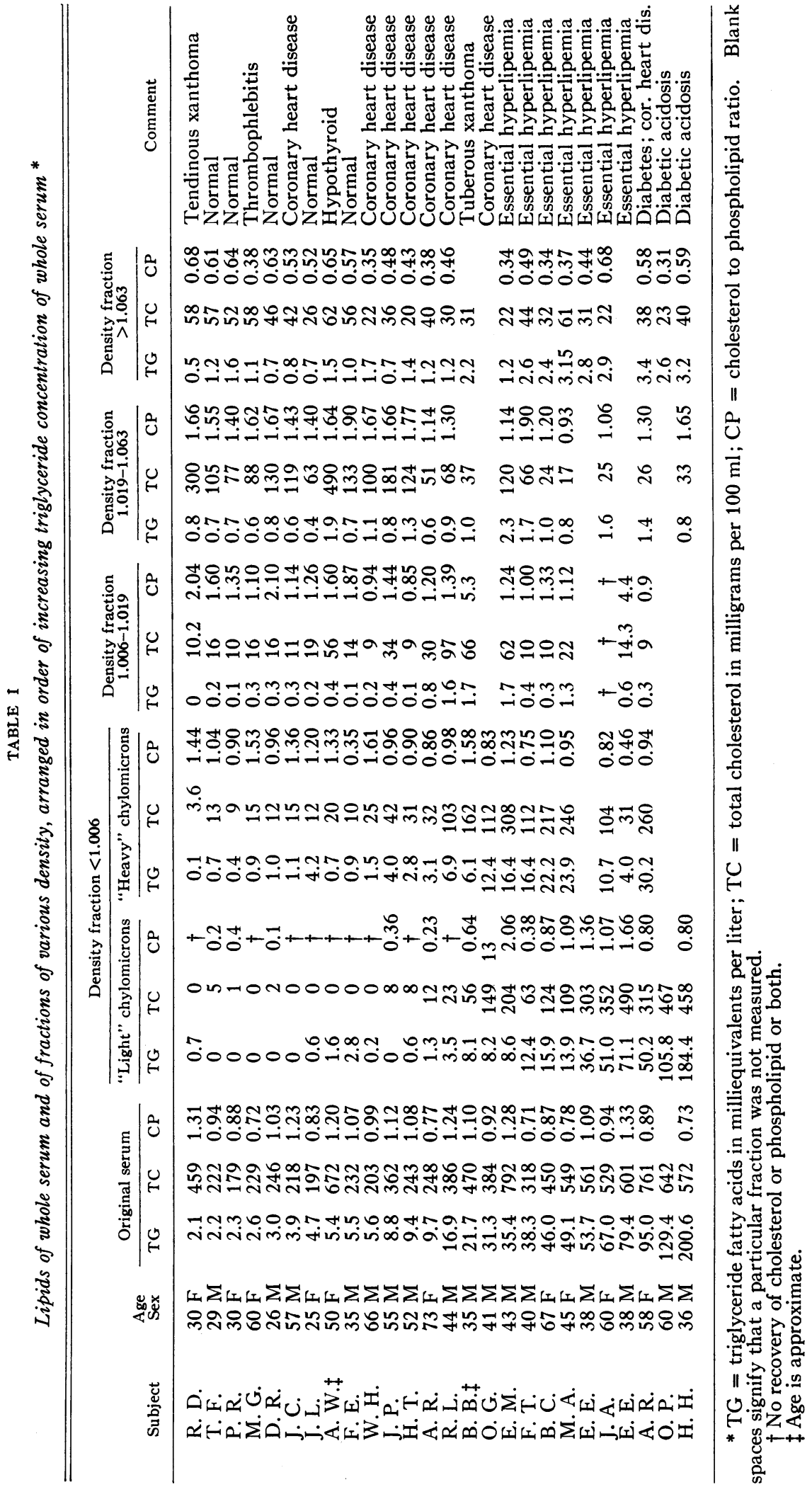


phospholipids about equally. Triglyceride recoveries were more variable, reflecting the greater error in their determination. In spite of the rather numerous opportunities for losses, a clear relationship between triglyceride concentration and lipoprotein pattern can be detected.

With increasing triglyceride concentration of whole serum, there were small increases in the triglycerides of the fractions $\mathrm{D}>1.006$, but the total triglyceride found in these three fractions together-D 1.006-1.019, 1.019-1.063, and > 1.063 -never exceeded $6 \mathrm{mEq}$ per $\mathrm{L}$. When total triglyceride concentration exceeded $6 \mathrm{mEq}$ per $\mathrm{L}$ the triglycerides in the "heavy" chylomicrons increased, but never above $30 \mathrm{mEq}$ per $\mathrm{L}$. The greatest triglyceride concentrations of very lipemic sera were found in the "light" chylomicrons, which contained as much as $184 \mathrm{mEq}$ per L, 90 per cent of the triglyceride in the most lactescent serum.

The cholesterol concentration in the most dense fraction, $D>1.063$, was fairly uniform throughout the entire group of sera, varying only between 20 and $62 \mathrm{mg}$ per $100 \mathrm{ml}$. There was an inconstant tendency for the cholesterol in this fraction to be lower in the lactescent than in the clear sera.

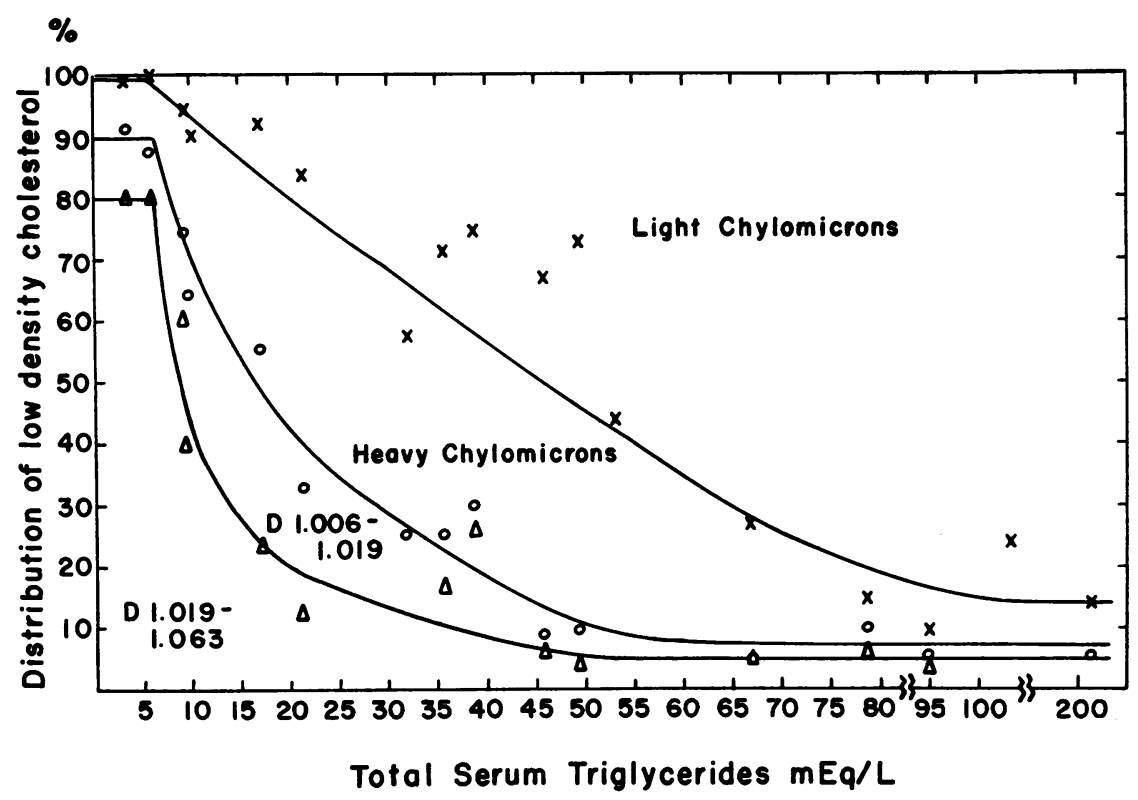

FIG. 1. EFFECT OF SERUM TRIGLYCERIDE CONCENTRATION, EXPRESSED AS TRIGLYCERIDE FATTY ACIDS, UPON THE DISTRIBUTION OF CHOLESTEROL AMONG LOW DENSITY LIPOPROTEIN FRACTIONS. The vertical axis represents the total cholesterol of $D<1.063$ in per cent. For each serum the distribution of cholesterol within the various low density subfractions is indicated by three symbols arranged vertically above the triglyceride concentration of that serum: triangles, D $>1.019$; circles, $\mathrm{D}>1.006$; X's, all the low density fractions except the easily removed "light" chylomicrons. The 100 per cent line represents all cholesterol of $\mathrm{D}<1.063$.

The first set of points (triglyceride concentration of $3.3 \mathrm{mEq}$ per $\mathrm{L}$ ) represents the average of the first eight sera from Table I. The second set of points (triglyceride concentration of $5.6 \mathrm{mEq}$ per $\mathrm{L}$ ) represents the average of the next two sera. The third set of points (triglyceride concentration of 9.3) represents the average of three patients whose triglyceride concentrations ranged from 8.8 to $9.7 \mathrm{mEq}$ per L. All other points represent individual sera. The lines were drawn to give the best visual fit to the various points. The mid-portion of line separating the "light" from the "heavy" chylomicrons was drawn to conform to the lower rather than the upper X's in recognition of the intermixture of these two layers during manipulation of the centrifuge tubes as a technical source of error, particularly at triglyceride concentrations of about $40 \mathrm{mEq}$ per $\mathrm{L}$. The vertical distances between the lines represent the relative cholesterol concentrations in any one category as indicated. 


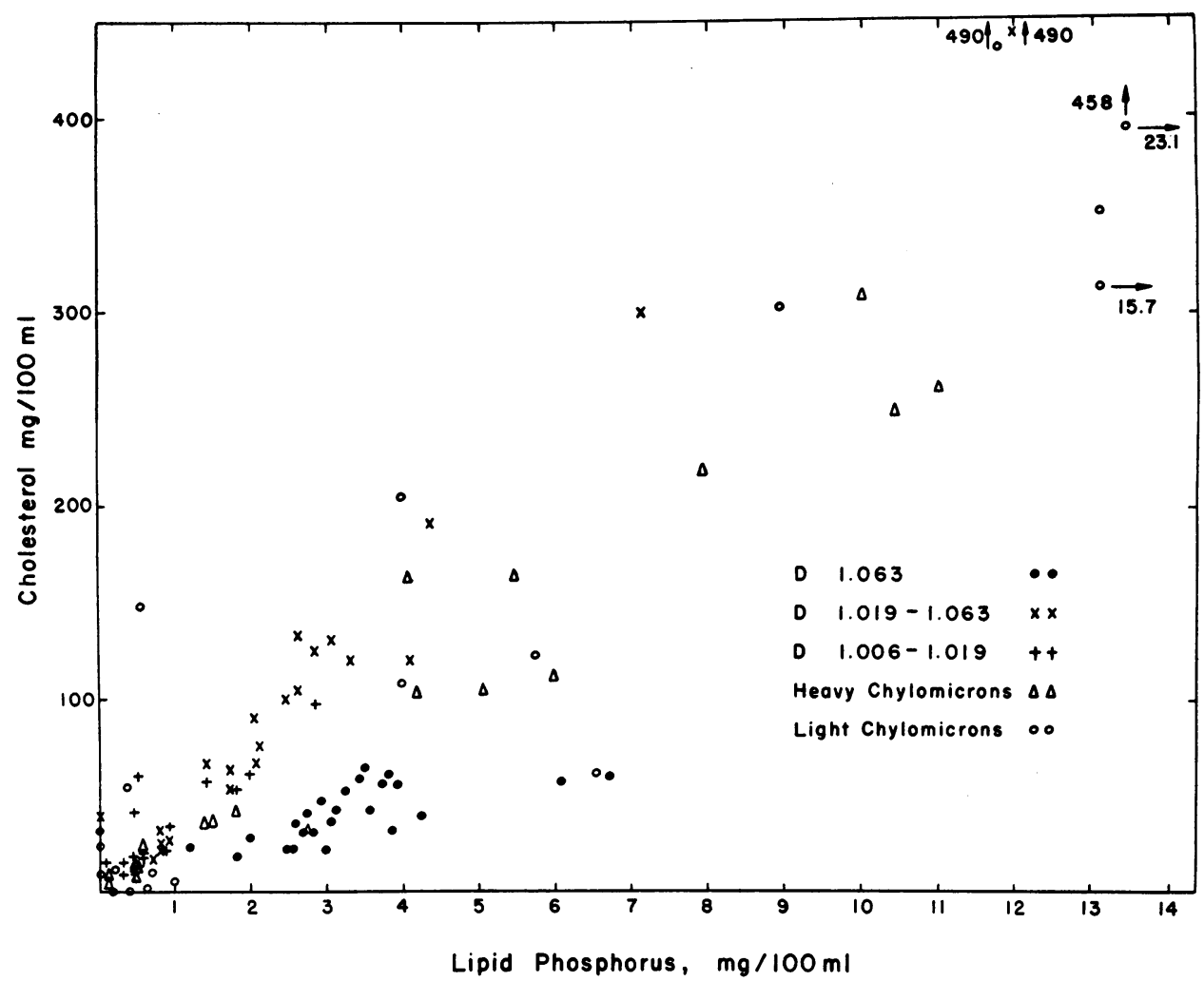

Fig. 2. ChOLESTEROL TO LIPID PHOSPHORUS RELATIONSHIP IN VARIOUS LIPOPROTEIN FRACTIONS IN A VARIETY OF NORMAL AND LIPEMIC SERA.

A remarkable influence of triglyceride concentration on the distribution of cholesterol among the various fractions of $\mathrm{D}<1.063$ is apparent in Table I. The cholesterol, which in the presence of normal triglycerides was largely concentrated in the fraction D 1.019-1.063, appeared, with increasing triglyceride concentration, in fractions of successively lower density. This relationship was even more prominent when the distribution of cholesterol among the low density fractions $(\mathrm{D}<1.063)$ was expressed as percentage of the total recovered low density cholesterol. Figure 1 shows the progressive shift of cholesterol from higher to intermediate density categories and, finally, out of the latter into the chylomicrons. In massively lactescent sera, 80 per cent or more of the cholesterol in all of the fractions of $\mathrm{D}<$ 1.063 was present in the "light" chylomicrons, leaving the intermediate density fractions relatively devoid of lipid.

The cholesterol to phospholipid ratio, calculated by using a factor of 25 for converting the lipid phosphorus into phospholipid, was charac- teristically low in the fraction of $\mathrm{D}>1.063$, ranging from 0.31 to 0.68 , tending to be a little lower in lactescent than in clear sera. The ratio was higher in the four lower density fractions, in most instances varying between 1.0 and 2.0. The low ratios found in the "light" chylomicrons of sera with normal total triglycerides cannot have much significance because of the great error of this ratio attendant upon the low concentrations of both cholesterol and phospholipid in this fraction. The cholesterol to phospholipid ratios in the various fractions are shown graphically in Figure 2. A rather constant linear relationship of cholesterol to lipid phosphorus is seen in the $D>1.063$ fraction, and a different but also rather constant relationship in the fractions of D 1.019-1.063 and 1.006-1.019. Between these two extremes are found most of the points representing the more variable cholesterol to phospholipid relationships of the fractions $\mathrm{D}<1.006$, the "heavy" and "light" chylomicrons.

Each serum was centrifuged in a single tube; the reproducibility of the methods of separation 
cannot therefore be determined from comparison of duplicate centrifugations but can be estimated by comparing results of different sera of similar triglyceride concentrations in this and a previous study (2). The "light" chylomicron fraction is uniformly of almost negligible lipid concentration in clear serum with normal triglyceride concentration, but is somewhat more variable in lactescent sera, as is evident from the scatter of the $\mathrm{X}$ 's about the line of best fit in Figure 1. In all fractions only 7 of 89 points, either shown individually in Figure 1 or used in obtaining the averaged points, departed from the appropriate line by more than 10 per cent on the vertical axis.

\section{DISCUSSION}

The present study shows the expected close relationship between total serum triglyceride concentration and lipoprotein pattern and permits the prediction of the latter from the concentration of total cholesterol and total triglyceride. The lipids were fractionated into those of high density, $\mathrm{D}>1.063$, and low density, $\mathrm{D}<1.063$. The latter were further divided into four subfractions. Although the concentration of low density cholesterol was related to the total serum cholesterol concentration, the relative distribution of low density cholesterol among the various subfractions appeared to be directly dependent upon serum triglyceride concentration. In the presence of normal triglycerides, the low density cholesterol and phospholipids were largely in the fraction D 1.019-1.063. With increased concentrations of total triglyceride, fatty acids above 6.0 $\mathrm{mEq}$ per $\mathrm{L}$ cholesterol and phospholipids were found in fractions of progressively lower density to an extent determined by the total triglyceride concentration. All increments of triglyceride above $6.0 \mathrm{mEq}$ per L occurred in the light-scattering fraction of $\mathrm{D}<1.006$.

Table II shows the percentage distribution of low density cholesterol among the four fractions of $\mathrm{D}<1.063$ at any given concentration of total triglyceride. The figures in this table were derived from the curves in Figure 1, which in turn were drawn as the lines visually best representing the individual points. By assuming a concentration of $40 \mathrm{mg}$ per $100 \mathrm{ml}$ for the cholesterol present in the fraction of $\mathrm{D}>1.063$, a fraction which
TABLE II

Distribution of cholesterol among low density lipoprotein fractions as a function of serum triglyceride concentration

\begin{tabular}{ccccc}
\hline \hline & \multicolumn{4}{c}{ Low density cholesterol } \\
\cline { 2 - 5 } $\begin{array}{c}\text { Triglyceride } \\
\text { fatty acids }\end{array}$ & \multicolumn{2}{c}{$<$ "Li.006* } & & \\
\cline { 2 - 5 }$m E q / L$ & $\%$ & $\%$ & $\%$ & $\%$ \\
5 & 1 & 9 & 10 & 80 \\
10 & 6 & 24 & 28 & 42 \\
20 & 20 & 38 & 22 & 20 \\
30 & 32 & 40 & 14 & 14 \\
40 & 43 & 38 & 10 & 9 \\
50 & 54 & 36 & 5 & 5 \\
60 & 65 & 27 & 3 & 5 \\
70 & 75 & 18 & 2 & 5 \\
80 & 80 & 13 & 2 & 5 \\
$100 \dagger$ & 86 & 7 & 2 & 5 \\
\hline
\end{tabular}

* Corresponds to $S_{f} 20-40,000$ (3).

+ Corresponds to $S_{\mathrm{f}} 12-20$.

$\mp$ Corresponds to $\mathrm{S}_{\mathrm{f}} \mathrm{O}-12$.

seems to be little if any influenced by triglyceride concentration, and by subtracting this figure from the total cholesterol, the concentration of cholesterol in the combined fractions of $\mathrm{D}<1.063 \mathrm{can}$ be estimated to within $20 \mathrm{mg}$ per $100 \mathrm{ml}$. If the triglyceride concentration is known, the absolute concentration of cholesterol in each of the four density fractions, which together comprise the group $\mathrm{D}<1.063$, can then be estimated from Table II.

The relationship between triglyceride concentration and lipoprotein pattern suggests a strong affinity between triglyceride-rich chylomicrons and lipoproteins of D 1.019-1.063. The inference from these data that cholesterol and phospholipids were removed from the D 1.019-1.063 fraction to lower density fractions is at least plausible. The exact nature of this affinity is not known, although the data from this and a previous paper (2) suggest a solubility distribution of cholesterol between an aqueous lipoprotein phase and a lipid chylomicron phase. Fredrickson and Gordon (14) have reviewed in detail evidence for affinity between both $\alpha$ - and $\beta$-lipoproteins and chylomicrons and the significance of this affinity in the clearing of chylomicrons from the circulation. The slight diminution of high density cholesterol in lipemic sera and the somewhat greater concentration of phospholipids relative to cholesterol in chylomicrons, as compared with lipoproteins of D 1.019-1.063, are consistent with the possible attraction of $\alpha$ - as well as $\beta$-lipoproteins to chylomi- 
crons. A fraction of serum cholesterol that is easily dissolved by organic solvents $(15,16)$ has been described-a phenomenon which might be used in support of the concept of a fraction of serum cholesterol easily dissolved in chylomicrons. The emulsifying action of phospholipids on fats is frequently mentioned, but the possible role of cholesterol in maintaining a fine state of emulsification of oils (17) is usually neglected. A chief function of lipoproteins may well be that of maintaining insoluble triglycerides in a state of fine emulsification and in a physical state promoting their interaction with a biochemical system that functions in aqueous solutions. The fine dispersion of the chylomicrons of even the most lipemic serum is impressive and difficult to create in artificial emulsions.

All of the lipemic sera of the present study must be considered abnormal in that the hypertriglyceridemia persisted for at least 14 hours after the last meal, usually sufficient time for the alimentary lipemia of the previous day to have subsided (18). The changes described here must be distinguished from the changes accompanying normal alimentary lipemia characterized by a small rise in triglyceride concentration restricted to the lipoproteins of $\mathrm{D}<$ $1.019(19,20)$, although an increase in the chylomicron cholesterol and an associated decrease in the cholesterol of the remaining serum may occur during the intravenous infusion of fat emulsions (21) in association with a much greater increase in triglyceride concentration than usually occurs after a fat meal.

The association between triglyceride concentration and lipoprotein pattern may have important implications in the field of coronary arterial disease. The presence of abnormally great concentrations of low density lipoproteins in sera of patients with coronary arterial disease, described by Gofman and colleagues (4), has been amply confirmed, but the separation between the normal and coronary populations on the basis of lipoproteins of the classes $S_{1} 12-20$ and $20-100$ has not proved to be very striking (22). A better separation was found by Gofman on the basis of the atherogenic index, an index based on a mathematical analysis of lipoproteins in which lipoproteins of the class $\mathrm{S}_{\mathrm{f}} 100-400$ are given relatively more weight than those of greater density (22). Since triglycerides constitute an increasingly important part of lipo- proteins of decreasing density, total triglyceride concentration provides an indication of lipoprotein concentration already weighted in favor of those of very low density, including those of $S_{f} 400$ and greater. Nichols, Lindgren and Gofman (23) have described a similar relationship between total lipid and atherogenic index. Total lipid, however, is composed largely of cholesterol and phospholipid in pure hypercholesterolemia, and largely of triglyceride in lipemic sera. There was little relationship between total cholesterol and its distribution among low density lipoproteins in the present study. Since total lipid is a rough indicator of triglyceride concentration, particularly in lipemic sera, Nichols and colleagues were probably in effect measuring the same relationship as that described here between triglyceride concentration and lipoprotein pattern.

Recent studies from this laboratory demonstrated that serum triglyceride fatty acid concentrations above $5.45 \mathrm{mEq}$ per $\mathrm{L}$ were characteristic of 80 per cent of a group of patients with coronary arterial disease $(6,7)$. This concentration was exceeded by only 5 per cent of young men of 20 to 29 years and by about 35 per cent of middle-aged men without known coronary arterial disease. Another communication contains a detailed comparison of the separation between a normal and coronary population, achieved on the basis of triglyceride concentration, with that effected on the basis of various categories of lipoproteins reported by others (7). It is perhaps more than coincidence that the concentration of triglyceride which best separates the normal from the coronary population coincides with the total maximal capacity of lipoproteins of D $>1.006$ for triglycerides, and with the concentration of triglyceride at which the shift of cholesterol from higher to lower density lipoproteins begins to occur.

Lipoprotein patterns have also been reported to be characteristic of disease states. Gofman, $\mathrm{Ru}$ bin, McGinley and Jones (24) noted that essential hyperlipemia and tuberous xanthoma were characterized by very low concentrations of lipoproteins $\mathrm{S}_{\mathrm{r}} \mathrm{O}-12$, corresponding to the density class $1.019-1.063$, but by great elevations of the classes $\mathrm{S}_{\mathrm{f}} 20-100$ and $100-400$ of density $<1.006$. In tendinous xanthomatosis there was an increase chiefly in the $\mathrm{S}_{\mathrm{r}} \mathrm{O}-12$ class. Nephrosis was asso- 
ciated with an increase in all lipoprotein densities up to $S_{f} 100-400$. These authors suggested that lipoprotein structure be considered the standard diagnostic feature of these diseases.

The relationships reported in the present paper are in accord with the findings of Gofman and associates. Patients with essential hyperlipemia as a rule have serum triglyceride concentrations great enough to be associated with a marked decrease of cholesterol in the fraction D 1.019-1.063 and an increase in the very low density cholesterol. On the other hand, the high cholesterol in the presence of normal triglycerides, characteristic of sera from patients with tendinous xanthomatosis, would be expected, on the basis of the present paper, to be concentrated in the fraction D 1.0191.063. Although no cases of nephrosis were included in the present study, Baxter, Goodman and Havel (25) reported that the lipoprotein pattern of nephrotic sera could be predicted from the triglyceride and cholesterol concentrations in a manner entirely consistent with the findings reported here.

On the basis of the present study it seems possible that the relative prominence of various categories of lipoproteins depends solely upon the total serum triglyceride concentration and thus upon the metabolism of triglyceride. The findings are in accord with the hypothesis that a delay in the normal rate of removal of triglycerides from the circulation, with a consequent rise in plasma triglyceride concentration, is responsible for the accumulation of very low density lipoproteins in plasma in certain diseases.

\section{SUM MARY}

Twenty-six clear and lactescent sera from normal persons and a variety of patients with disorders of lipid metabolism were analyzed for cholesterol, triglycerides, and cholesterol to phospholipid ratio of whole serum and of various lipoprotein fractions. Lipoproteins were ultracentrifugally separated into fractions of density (D) $>$ 1.063, D 1.019-1.063, D 1.006-1.019 and D < 1.006. The latter were further divided into "heavy" and "light" chylomicrons.

Triglyceride concentration of whole serum had a marked effect upon lipoprotein pattern. All triglyceride fatty acid increments over $6 \mathrm{mEq}$ per
$\mathrm{L}$ occurred in the fraction $\mathrm{D}<1.006$. As triglycerides increased above this concentration, the relative concentration of cholesterol among the various fractions of $\mathrm{D}<1.063$ shifted out of the fraction D 1.019-1.063 and into fractions of progressively lower density. On the basis of data presented, it was possible to predict the cholesterol concentration of the various lipoprotein fractions from the concentration of triglycerides and cholesterol of whole serum.

\section{REFERENCES}

1. Mariet, cited by Thannhauser, S. J. Lipidoses: Diseases of the cellular lipid metabolism in Oxford Medicine. Oxford, Oxford Univ. Press, 1949, vol. IV, part II, chap. VII, p. 214.

2. Albrink, M. J., Man, E. B., and Peters, J. P. The relation of neutral fat to lactescence of serum. J. clin. Invest. 1955, 34, 147.

3. Eder, H. A. The lipoproteins of human serum. Amer. J. Med. 1957, 23, 269.

4. Gofman, J. W., Lindgren, F., Elliott, H., Mantz, W., Hewitt, J., Strisower, B., and Herring, V. The role of lipids and lipoproteins in atherosclerosis. Science 1950, 111, 166.

5. Man, E. B., and Albrink, M. J. The significance of triglycerides of fatty acids in biological fluids in Blood Lipids and the Clearing Factor. Third Internat. Conf. on Biochemical Problems of Lipids, Brussels, July, 1956, p. 200.

6. Albrink, M. J., and Man, E. B. Serum triglycerides in coronary artery disease. A. M. A. Arch. intern. Med. 1959, 103, 4.

7. Albrink, M. J., Meigs, J. W., and Man, E. B. Serum lipids, hypertension and coronary artery disease. Amer. J. Med. In press.

8. Antonis, A., and Bersohn, I. Serum-triglyceride levels in South African Europeans and Bantu and in ischaemic heart-disease. Lancet 1960, 1, 998.

9. Havel, R. J., Eder, H. A., and Bragdon, J. H. The distribution and chemical composition of ultracentrifugally separated lipoproteins in human serum. J. clin. Invest. 1955, 34, 1345.

10. Albrink, M. J. The microtitration of total fatty acids of serum, with notes on the estimation of triglycerides. J. Lip. Res. 1959, 1, 53.

11. Abell, L. L., Levy, B. B., Brodie, B. B., and Kendall, F. E. A simplified method for the estimation of total cholesterol in serum and demonstration of its specificity. J. biol. Chem. 1952, 195, 357.

12. Fiske, C. H., and Subbarow, Y. The colorimetric determination of phosphorus. J. biol. Chem. 1925, 66, 375.

13. Peters, J. P., and Man, E. B. The interrelations of serum lipids in normal persons. J. clin. Invest. 1943, 22, 707. 
14. Fredrickson, D. S., and Gordon, R. S., Jr. Transport of fatty acids. Physiol. Rev. 1958, 38, 585.

15. Taylor, P. C., Forbes, J. C., and Petterson, O. M. Effect of lyophilization and chloroform extraction on electrophoretic migration of serum lipoproteins. Proc. Soc. exp. Biol. (N. Y.) 1956, 91, 562.

16. Byers, S. O., and Friedman, M. The fractionation of cholesterol in body fluids by means of solvent extraction. J. clin. Invest. 1956, 35, 405.

17. Schulman, J. H., and Cockbain, E. G. Molecular interaction at oil-water interfaces. I. Molecular complex formation and the stability of oil-in-water emulsions. Faraday Soc. Trans. 1940, 36, 651.

18. Kuo, P. T., and Carson, J. C. Dietary fats and the diurnal serum triglyceride levels in man. J. clin. Invest. 1959, 38, 1384.

19. Havel, R. J. Early effects of fat ingestion on lipids and lipoproteins of serum in man. J. clin. Invest. 1957, 36, 848.

20. Havel, R. J., and Fredrickson, D. S. The metabolism of chylomicra. I. The removal of palmitic
acid-1-C ${ }^{14}$-labeled chylomicra from dog plasma. J. clin. Invest. 1956, 35, 1025.

21. Albrink, M. J., and Man, E. B. Serum triglycerides in health and diabetes. Diabetes 1958, 7, 194.

22. The Technical Group of the Committee on Lipoprotein and Atherosclerosis, and the Committee on Lipoproteins and Atherosclerosis of the National Advisory Heart Council. Evaluation of serum lipoprotein and cholesterol measurements as predictors of clinical complications of atherosclerosis. Circulation 1956, 14, 691.

23. Nichols, A. V., Lindgren, F. T., and Gofman, J. W. Estimation of atherogenic index and lipoprotein distribution in man. Evaluation from serum gravimetric total lipid and total cholesterol concentration. Geriatrics 1957, 12, 130.

24. Gofman, J. W., Rubin, L., McGinley, J. P., and Jones, H. B. Hyperlipoproteinemia. Amer. J. Med. 1954, 17, 514.

25. Baxter, J. H., Goodman, H. C., and Havel, R. J. Serum lipid and lipoprotein alterations in nephrosis. J. clin. Invest. 1960, 39, 455. 